\title{
High Gain and Wide Band Antenna Based on FSS and RIS Configuration
}

\author{
Guthi SRINIVAS, Damera VAKULA
}

Dept. of Electronics and Communication Engineering, National Inst. of Technology Warangal, 506 004, Telangana, India

guthisrinivas37@gmail.com, vakula@nitw.ac.in

Submitted October 27, 2020 / Accepted December 20, 2020

\begin{abstract}
In this paper, a novel technique using frequency selective surface (FSS) superstrate is proposed to increase the antenna gain. In addition to that, a combination of reactive impedance surfaces (RIS) is included to enhance the bandwidth. So, a conventional pentagon shape patch antenna is designed at $5.3 \mathrm{GHz}$. The frequency selective surface is designed as a $6 \times 6$ array of unit cell structures to operate around $5.3 \mathrm{GHz}$. Each unit cell consists of three metal conductive layers with substrates in between them. Reactive Impedance Surface is considered as an array size of $6 \times 6$ square patches embedded between two substrates. FSS is designed on Rogers 4003C and FR4 is used for RIS. The pentagon shape patch antenna is designed on RIS. A cavity created by the contribution of these layers acts like a Fabry Perot resonator which improves the gain and bandwidth simultaneously. The proposed antenna has an impedance bandwidth of 17.72\% (4.93-5.89 GHz); this is about a 10 percent improvement over the impedance bandwidth of a conventional pentagon shape antenna and the axial ratio bandwidth is $2.4 \%(5.01-5.14 \mathrm{GHz})$. The designed antenna gain is around $12 \mathrm{dBi}$; this is about a 9dBi improvement over the gain of a conventional pentagon shape antenna.
\end{abstract}

\section{Keywords}

Pentagon patch antenna, circular polarization frequency selective surface superstrate, reactive impedance surface, Fabry-Perot resonator, gain, bandwidth improvement

\section{Introduction}

Nowadays, many applications such as high definition media devices are developed at $5 \mathrm{GHz} \mathrm{Wi}-\mathrm{Fi}$ and Wi-Max frequency band. These applications require high gain, wideband and directional antennas. The gain of the antenna should be high which consumes less power and can be transmitted to long distances. Impedance bandwidth should be more to transmit entire frequency bands maintaining good radiation patterns in broadside direction. The microstrip patch antennas are resonant antennas with narrow bandwidth which radiates along broadside direction with a directional radiation pattern. The circular polarization (CP) is preferred over linear polarization (LP) because the signal transmission is possible irrespective of the orientation of the transmitting and receiving antenna. The LP waves change the polarization in the atmosphere due to Faradays rotation, whereas $\mathrm{CP}$ waves maintain the same polarization in the atmosphere.

Several techniques for the gain enhancement are already reported in the published literature: antenna arrays principle, antenna hybridization, and surface-mounted horn antenna. One of the best techniques is antenna array [1-4], where several antennas are used to increase the gain. However, cost and complexity in the feeding network are major problems in the antenna array. Fabry-Perot Resonator (FPR) antennas are one of the recent techniques developed to improve antenna performance with less cost and complexity. In the FPR, a cavity is created between the ground plane and the superstrate. There are different types of superstrates used such as partially reflecting surface, frequency selective surface, metamaterials, etc. Robert Orr et al. published a Fabry Perot resonator antenna which consists of HIS surface and a partially reflecting surface to get high gain. The square patch is rotated by 45 degrees to get circular polarization [5]. Kamil Pitra et al. proposed the bottom wall of the Fabry Perot antenna with a mushroomlike EBG surface and the top wall of the Fabry Perot antenna with a partially reflecting surface to improve the gain of the antenna. The CP FPR is created by tilting the patch by 45 degrees and the cross slot is chosen in the ground plane [6]. The magnetoelectric dipole antenna is designed to get wideband circular polarization which is introduced by Wenquan Cao et al. This antenna is used as a feeding element along with different types of FSS structures and also compared. The use of circular shape in FSS superstrate is well illustrated to get high CP gain [7]. The cross slot coupled DRA element as feeding element and FSS as superstrate is proposed by M. Akbari et al. to design a high gain Fabry Perot antenna. The cross slot is chosen to get circular polarization [8]. Hussein Attia et al. proposed a partially reflecting surface with two layers of frequency selective surface superstrate, which consists of a gridded square patch and a square slot loaded patch on the bottom faces of the top and bottom substrates respectively. The slot 
antenna with the above-mentioned superstate improves the gain of the antenna [9]. Yuejun Zheng et al. proposed a Chess Board Arranged Metamaterial Superstrate made up of two kinds of frequency selective surface at $10.8 \mathrm{GHz}$ to achieve improvement in gain [10]. Muftah Asaadi published a frequency selective superstrate layer placed above the high dense dielectric patch with relative permittivity 82 to achieve high gain [11]. M. A. Meriche et al. suggested a single layer metallic superstrate as an FSS along with the excitation antenna to improve the gain of the antenna [12]. Fabry Perot cavity is implemented to improve the gain of the antenna, where the analysis is done by changing the dimensions of FSS unit cell and an array of unit cells in FSS [13], [14]. The above-mentioned techniques can improve the gain of the antenna without any increase in impedance bandwidth.

Several techniques for bandwidth improvement are reported in literature like stacked patches and parasitic elements. These parasitic elements are used on the entire surface to get better impedance bandwidth. Impedance matching networks are used with L-shaped probes and aperture coupling to get circularly polarized antenna [15-16]. A wideband antenna is obtained by using the air gap between the patches and circular polarization is achieved using truncated corners [17]. These structures are using large antenna heights which increase the volume of the antenna. Consequently, high impedance electromagnetic surfaces [18] and artificial ground structures are used [19] to solve these problems. Artificial ground structures with a truncated corner square patch are proposed by Teruhisa Nakamura et al. to get circular polarization and to improve the impedance bandwidth [20]. These techniques are focusing only to improve impedance bandwidth without any increase in gain. The authors have published a combination of the conventional antenna along with reactive impedance surface and frequency selective surface to improve the gain and bandwidth simultaneously [21]. The superstrate is designed with square patches, but the use of square patches in the superstrate reduces the $\mathrm{CP}$ gain due to its non-symmetry property along $\varphi$ direction [7]. In this paper, the circle-shaped patches are used in the superstrate as it shows symmetry property along $\varphi$ direction and modified fishnet structure is used to get improvement in the CP gain over a wide frequency range.

This paper presents a high gain and wideband antenna, which is composed of a pentagon patch antenna, Frequency Selective Surface (FSS), and Reactive Impedance Surface (RIS). The combination of a conventional antenna with FSS and RIS acts like a Fabry Perot cavity resonator which improves the gain and bandwidth simultaneously as compared with the conventional antenna. The antenna design is simulated using High-Frequency Structure Simulator and measurement results are done in an anechoic chamber. It is demonstrated that measured results are almost similar to simulated results.

This paper is organized as follows. In Sec. 2, the conventional antenna design is discussed. In Sec. 3, the antenna with RIS configuration is explained. In Sec. 4, the

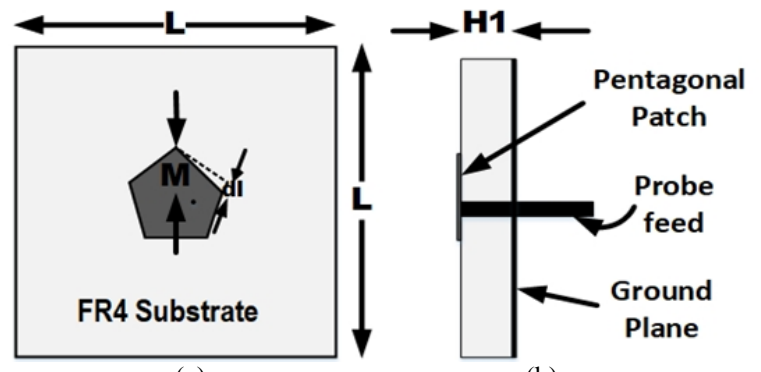

(a)

(b)

Fig. 1. The conventional pentagon shape patch antenna: (a) Top view. b) Side view.

unit-cell design and characteristics are explained. In Sec. 5, the antenna with FSS and RIS configuration and its results are demonstrated. In Sec. 6, antenna fabrication and measurement results are compared with simulated results.

\section{Pentagon Shape Patch Antenna}

Pentagon shape patch antenna provides better performance than other conventional structures like square, circle, and rectangular structures. Other conventional structures need multiple feeding to get circular polarization whereas pentagon shape patch antenna generates circular polarization with single feeding only. The equilateral pentagon antenna could not generate circular polarization. A small portion of the right side edge is removed from the equilateral pentagon shape antenna as shown in Fig. 1 [22]. The feed position of the antenna is optimized to generate two orthogonal fields perpendicular to each other i.e. circular polarization. The pentagon shape patch is printed above the FR4 substrate and the metal layer in the bottom acts as a ground. The probe feeding is given by the SMA connector. The inner connector of SMA is connected to the pentagon patch and the outer part of SMA is connected to the ground.

This antenna design is based on the finite element method in High-Frequency Structure Simulator software. The simulation results show that impedance bandwidth is $7.8 \%(5.11-5.52 \mathrm{GHz})$, gain is $3.4-3.5 \mathrm{dBi}$, and axial ratio is $1.9 \%(5.27-5.37 \mathrm{GHz})$ as shown in Fig. $5 \mathrm{a}, \mathrm{b}$ and c, respectively. The substrate is considered large enough to accommodate the modification done for gain and bandwidth.

\section{Pentagon Shape Patch Antenna on RIS Structure}

A reactive impedance surface is a surface that introduces additional reactance to improve the matching. In the proposed pentagon antenna RIS surface is added in between the patch and ground plane to improve the impedance bandwidth of the antenna [20]. An array of patches will be introduced between the patch and ground plane and for the proposed design, square patches are selected. The size of the patch and the number of patches are the param- 
eters that control the matching. After optimizing the patch size and array size, the array is made by a $6 \times 6$ array of square patches printed on the top side of the FR4 substrate. The parametric study is done on the dimension of the square patch $(L 1)$, its periodicity $(L 1+p)$, substrate height (H1) below the RIS surface, and substrate height $(H 2)$ above the RIS surface. The side of square patches $(L 1)$, the distance between the patches $(p)$, the height $H 1$, and the height $H 2$ are optimized for wide bandwidth to $4.5 \mathrm{~mm}$, $5.5 \mathrm{~mm}, 1.6 \mathrm{~mm}$, and $0.8 \mathrm{~mm}$, respectively. After optimization of the RIS structure, an impedance bandwidth of $12.2 \%(5.05 \mathrm{GHz}-5.71 \mathrm{GHz})$ is achieved as shown in Fig. $5 \mathrm{a}$, and also there is an increase in the $3 \mathrm{~dB}$ axial ratio bandwidth. An axial ratio bandwidth of $2.7 \%(5.21 \mathrm{GHz}$ to $5.35 \mathrm{GHz}$ ) is achieved as shown in Fig. 5c. The RIS technique has improved the bandwidth, but there is no considerable improvement in the antenna gain as shown in Fig. 5b.

\section{Frequency Selective Surface (FSS) Superstrate}

FSS is a surface in which the transmission and reflection characteristics vary with frequency. The transmission is varied by using an array of unit cells. The surface contains a periodic array of unit cells printed on one side or two sides of the substrate. To modify the wave propagation, the FSS is placed along the direction of wave propagation. The superstrate acts like a lens above the antenna and it will improve the gain of the antenna when constructive interference is created between the antenna and superstrate.

\subsection{Highly Reflective Unit Cell Design}

The design details of highly reflective unit cells are studied in this section. The circle-shaped patches are preferred in FSS superstrate due to their symmetry nature along the $\varphi$ direction and improve the $\mathrm{CP}$ gain over the wide frequency range [7]. The highly reflective unit cell consists of circle-shaped patches on the top layer and bottom layer. In addition to that, a modified fishnet structure is used as a middle layer. The three layers are separated by the substrate in between them as shown in Fig. 2a. The top and bottom layers act like the capacitive layer and the middle layer acts as an inductive layer to form a superstrate.

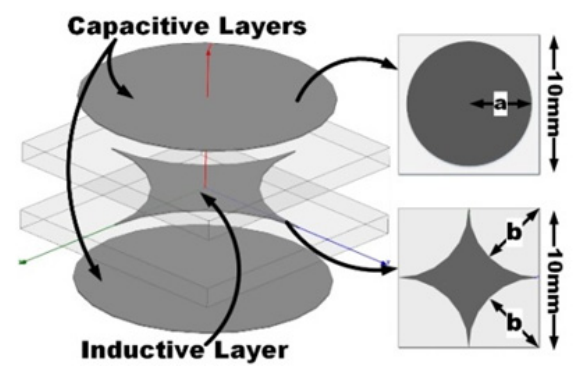

Fig. 2a. Three-dimensional view of highly reflective FSS unit cell.

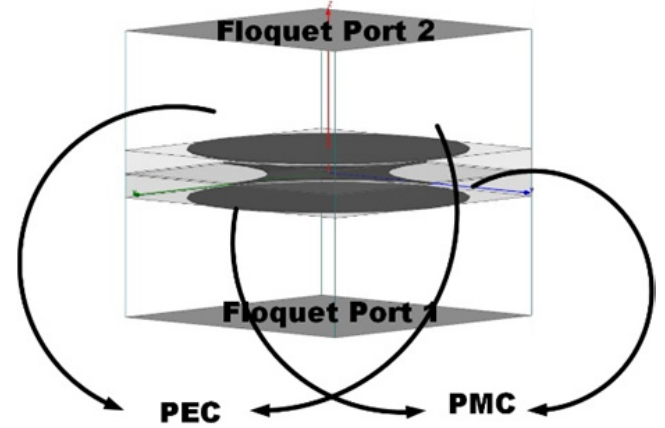

Fig. 2b. Boundary conditions of a highly reflective FSS unit cell.

The highly reflective frequency selective surface is designed as a $6 \times 6$ array of periodic unit cells printed on Rogers's 4003C substrate, which is used as a superstrate of the antenna. When the FSS superstrate is placed at an approximately half-wavelength height from the antenna, it creates an air-filled cavity between the superstrate and ground plane. The electromagnetic rays are focussed along the transmit direction and form concentrated rays in the broadside direction, thus, the gain of an antenna is improved, when a highly reflective superstrate is placed at an optimum height from the antenna. It is well known that directivity can be enhanced as the magnitude of the reflection coefficient increases to unity $(0 \mathrm{~dB})$.

The unit cell is designed in HFSS software and boundary conditions are applied as shown in Fig. $2 b$. The parametric study is done by changing $a$ and $b$ values, where $a$ is the radius of the top side conductor and $b$ is the curvature radius of the bottom conductor. These dimensions of the

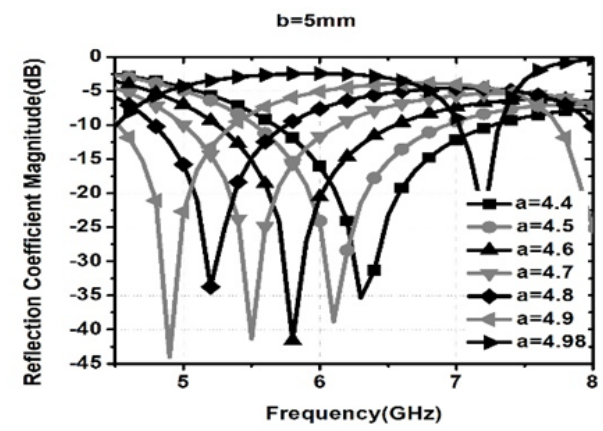

Fig. 3a. Magnitude of reflection coefficient for highly reflective FSS unitcell.

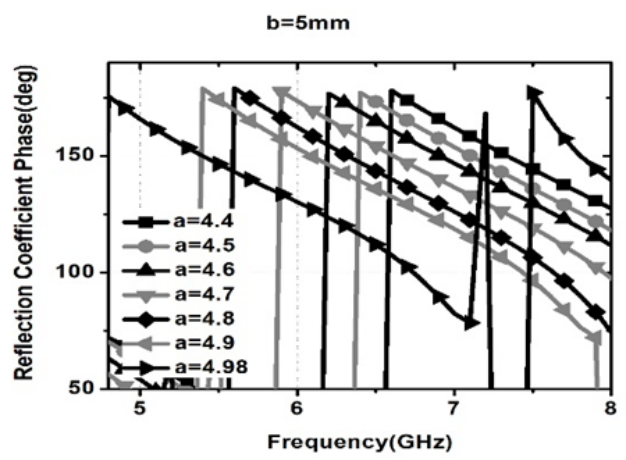

Fig. 3b. Phase of reflection coefficient for highly reflective FSS unit cell. 
unit cell control the characteristics of FSS. The magnitude and phase of the reflection coefficient are shifted to the lower frequencies with an increase in the radius $a$ as shown in Fig. 3. The magnitude of the reflection coefficient increases towards $0 \mathrm{~dB}$ i.e. unity in the selected frequency range $(5.1 \mathrm{GHz}-5.7 \mathrm{GHz})$ and the phase of the reflection coefficient is near $180^{\circ}$ over the selected frequency range $(5.1 \mathrm{GHz}-5.7 \mathrm{GHz})$ as dictated by the requirements of a highly reflective superstrate to improve gain. These conditions are achieved when $a=4.98 \mathrm{~mm}$ and $b=5 \mathrm{~mm}$. The optimized dimensions of antenna and unit cell dimensions are given in Tab. 1.

\section{Antenna with RIS - FSS Configuration}

The proposed antenna is composed of the pentagon shape patch antenna along with highly reflective FSS and RIS structures. RIS structure is attached beneath the pentagon shape patch antenna and highly reflective FSS is positioned above the antenna as shown in Fig. 4a and Fig. 4b. The cavity created by the contribution of these layers acts like a Fabry Perot cavity resonator. Here, the directly transmitted rays from the antenna and reflected rays from the superstrate trapped in the cavity. The coherent addition of trapped rays radiates along broadside direction. Hence, the gain of the antenna improved remarkably.

The proposed antenna with RIS and FSS is simulated in HFSS software. The simulated results are shown in Fig. 5.

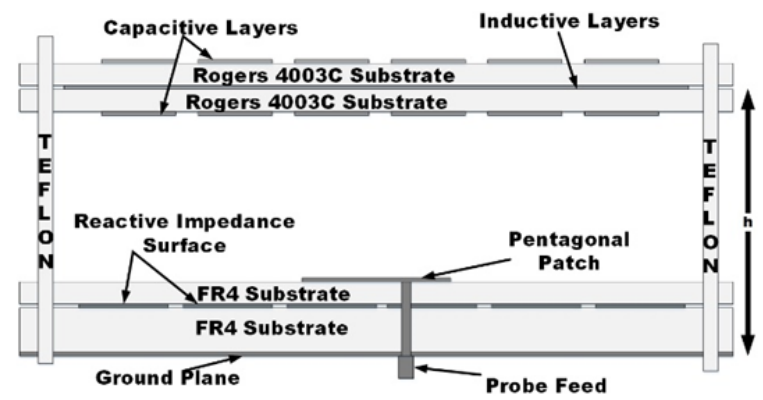

Fig. 4a. Geometry of composite structure of pentagon antenna with RIS and FSS (Front View).

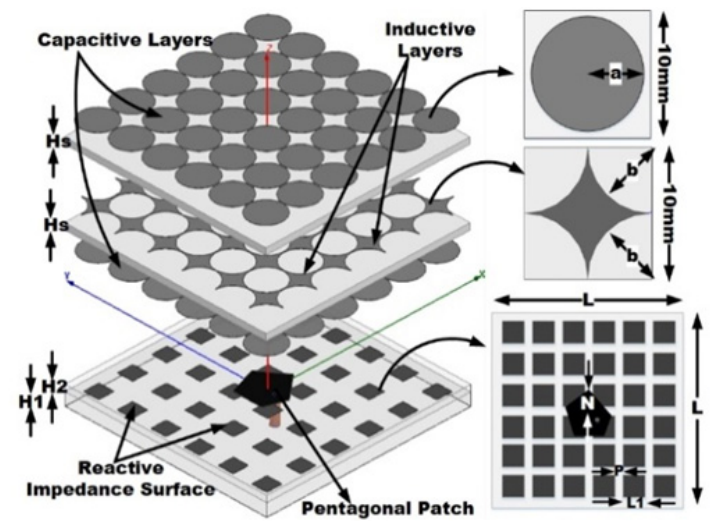

Fig. 4b. Three-dimensional view of a composite structure of pentagon antenna with RIS and FSS.

\begin{tabular}{|c|c|}
\hline Parameter & Value(mm) \\
\hline$L$ & 60 \\
\hline$H 1$ & 1.6 \\
\hline$H 2$ & 0.8 \\
\hline$H s$ & 0.813 \\
\hline$H s$ & 0.813 \\
\hline$M$ & 8.4 \\
\hline$N$ & 7.5 \\
\hline$d l$ & 1.7 \\
\hline$L 1$ & 4.5 \\
\hline$p$ & 5.5 \\
\hline$a$ & 4.98 \\
\hline$b$ & 5 \\
\hline$h$ & 32 \\
\hline
\end{tabular}

Tab. 1. Parameter values.

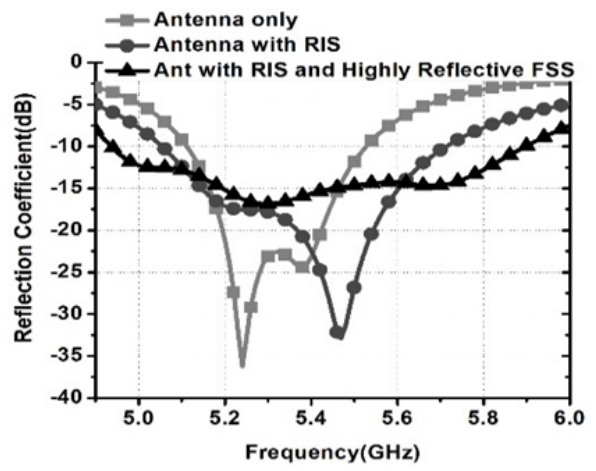

Fig. 5a. Simulated S11 of antenna, antenna with RIS, and antenna with RIS and FSS at $h=32 \mathrm{~mm}$.

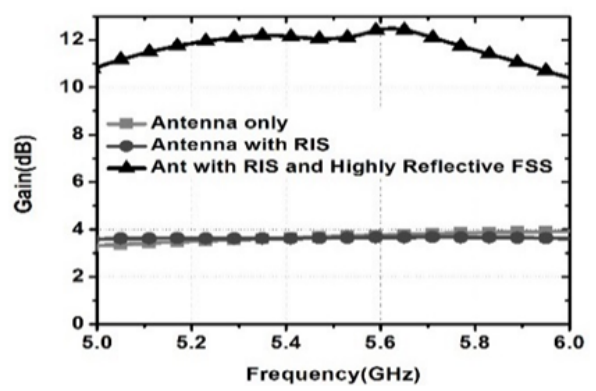

Fig. 5b. Simulated gain of antenna, antenna with RIS, and antenna with RIS and FSS at $h=32 \mathrm{~mm}$.

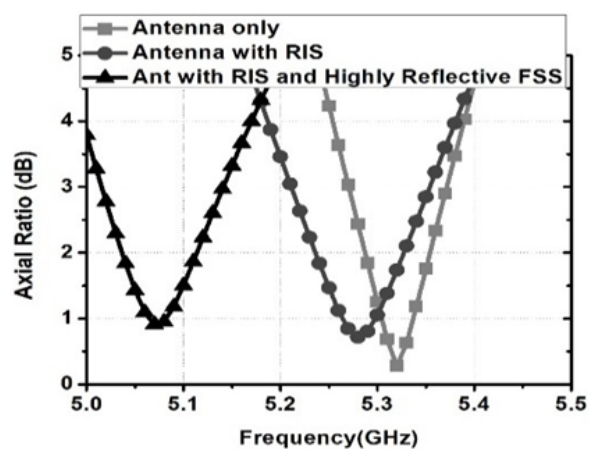

Fig. 5c. Simulated axial ratio of antenna, antenna with RIS, and antenna with RIS and FSS at $h=32 \mathrm{~mm}$.

The impedance bandwidth is $17.72 \%$ as shown in Fig. 5a and the improvement is around 10\%. The antenna gain is around $12 \mathrm{dBi}$ in the frequency range of $5.1 \mathrm{GHz}$ to $5.7 \mathrm{GHz}$ as shown in Fig. $5 \mathrm{~b}$ and the improvement in the 
gain is around $9 \mathrm{dBi}$. The axial ratio is $2.5 \%$ as shown in Fig. 5c. The proposed antenna results are compared with basic conventional antenna results and its results are mentioned in Tab. 2.

It is observed that the conventional antenna with RIS structure has considerable improvement in the impedance bandwidth as shown in Fig. 5a. But there are no considerable improvements in the antenna gain as shown in Fig. 5b. The conventional antenna with RIS structure and FSS superstrate has improvement in both gain and impedance bandwidth simultaneously as shown in Fig. 5a and Fig. 5b. Besides, the antenna has a $3 \mathrm{~dB}$ axial ratio bandwidth of $2.4 \%$ as shown in Fig. $5 \mathrm{c}$.

It is observed that the proposed antenna surface current density vector on the patch is rotating clockwise direction as the phase increases i.e the antenna is left hand circularly polarized (LHCP) as the direction of propagation $(Z)$ is topside antenna as shown in Fig. 6. The surface current density vector is towards 135 degrees for the phase 0 degree as shown in Fig. $6 \mathrm{a}$, it is towards 45 degrees for the phase 90 degrees as shown in Fig. 6 b, it is towards -45 degrees for the phase 180 degrees as shown in Fig. 6c, it is towards -135 degrees for the phase 270 degrees as shown in Fig. 6d.

The LHCP gain is high over the entire bandwidth as shown in Fig. 7. The right hand circularly polarized (RHCP) gain is $-15 \mathrm{~dB}$ at $5.1 \mathrm{GHz}$ frequency where the antenna is maintaining circular polarization and the RHCP gain is increasing in other frequencies as shown in Fig. 7.

\begin{tabular}{|c|c|c|c|c|}
\hline $\begin{array}{c}\text { S. } \\
\text { No. }\end{array}$ & Antenna & $\begin{array}{c}\text { Impedance } \\
\text { Bandwidth }\end{array}$ & $\begin{array}{c}\text { Gain } \\
\text { (dBi) }\end{array}$ & Axial Ratio \\
\hline 1 & $\begin{array}{c}\text { Pentagon } \\
\text { Shape Patch } \\
\text { Antenna }\end{array}$ & $\begin{array}{c}7.8 \% \\
(5.11-5.52 \mathrm{GHz})\end{array}$ & $3.4-3.7$ & $\begin{array}{c}1.90 \% \\
(5.27-5.37 \mathrm{GHz})\end{array}$ \\
\hline 2 & $\begin{array}{c}\text { Pentagon } \\
\text { Shape with } \\
\text { RIS }\end{array}$ & $\begin{array}{c}12.24 \% \\
(5.05-5.71 \mathrm{GHz})\end{array}$ & $3-4$ & $\begin{array}{c}2.7 \% \\
(5.21-5.35 \mathrm{GHz})\end{array}$ \\
\hline 3 & $\begin{array}{c}\text { Pentagon with } \\
\text { RIS and highly } \\
\text { reflective FSS }\end{array}$ & $\begin{array}{c}17.72 \% \\
(4.93-5.89 \mathrm{GHz})\end{array}$ & 12.48 & $\begin{array}{c}2.4 \% \\
(5.01-5.14 \mathrm{GHz})\end{array}$ \\
\hline
\end{tabular}

Tab. 2. Comparison of antenna, antenna with RIS, and antenna with RIS and FSS at $h=32 \mathrm{~mm}$.

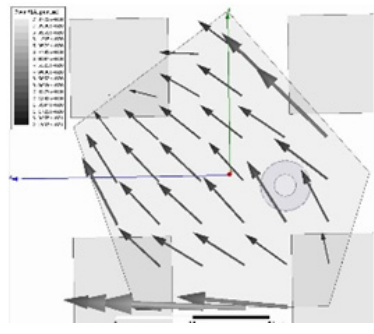

a. Phase $=0$ degree

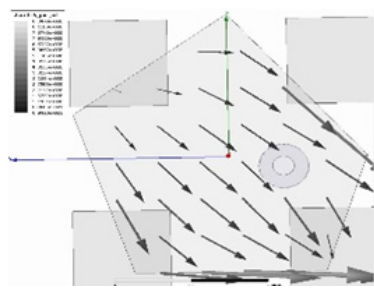

c. Phase $=180$ degree

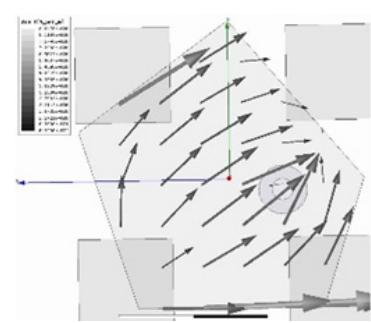

b. Phase $=90$ degree

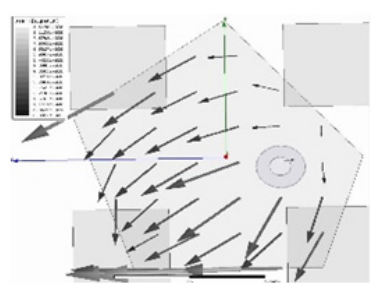

d. Phase $=270$ degree
Fig. 6. Antenna with RIS and FSS surface current density vector on pentagon patch at $5.1 \mathrm{GHz}$.

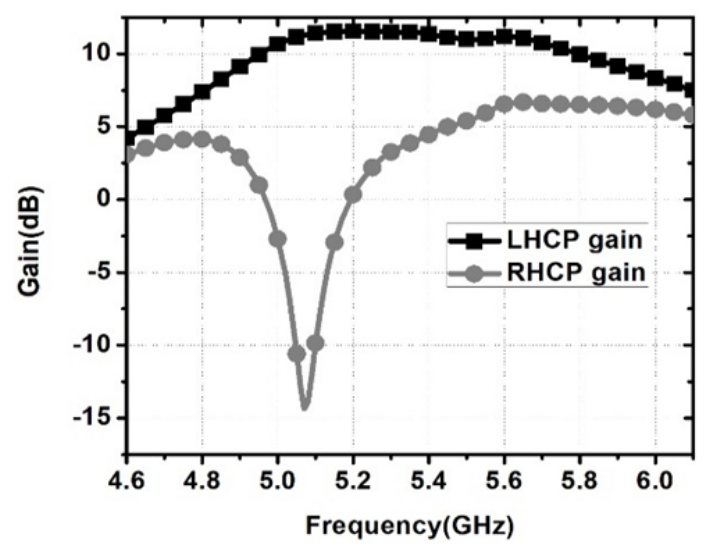

Fig. 7. Simulated LHCP and RHCP gain plot for the antenna with RIS and FSS.

The proposed antenna results are compared with published literature as shown in Tab. 3. It is observed that the proposed antenna planar size is very less $\left(1 \lambda_{\mathrm{o}} \times 1 \lambda_{\mathrm{o}}\right)$ and the superstrate is placed approximately half-wavelength height $\left(\lambda_{\mathrm{o}} / 2\right)$ as mentioned in published literature.

\begin{tabular}{|c|c|c|c|c|c|c|c|}
\hline S. No. & Ref & $\begin{array}{c}\text { Frequency range } \\
(\mathbf{G H z})\end{array}$ & $\begin{array}{c}\text { Planar Size } \\
(\mathbf{m m} \times \mathbf{m m})\end{array}$ & $\begin{array}{c}\text { Height of } \\
\text { Superstrate (mm) }\end{array}$ & Gain(dBi) & $\begin{array}{c}\text { Impedance } \\
\text { Bandwidth (\%) }\end{array}$ & Axial Ratio (\%) \\
\hline 1 & {$[5]$} & $14.9-15.3$ & $7 \lambda_{\mathrm{o}} \times 7 \lambda_{\mathrm{o}}$ & $\approx \lambda_{\mathrm{o}} / 2$ & $21 \mathrm{dBi}$ & $2.5 \%$ & $7 \%$ \\
\hline 2 & {$[6]$} & $9.85-10.1$ & $2.758 \lambda_{\mathrm{o}} \times 2.758 \lambda_{\mathrm{o}}$ & $\approx \lambda_{\mathrm{o}} / 2$ & $15 \mathrm{dBi}$ & $2.3 \%$ & $0.6 \%$ \\
\hline 3 & {$[7]$} & $11.7-19.8$ & $1.75 \lambda_{\mathrm{o}} \times 1.75 \lambda_{\mathrm{o}}$ & $\approx \lambda_{\mathrm{o}} / 2$ & $11.5 \mathrm{dBi}$ & $54 \%$ & $29 \%$ \\
\hline 4 & {$[8]$} & $29.1-31.6$ & $2.5 \lambda_{\mathrm{o}} \times 2.5 \lambda_{\mathrm{o}}$ & $\approx \lambda_{\mathrm{o}} / 2$ & $15.2 \mathrm{dBi}$ & $9.6 \%$ & $2.67 \%$ \\
\hline 5 & {$[9]$} & $55.4-66.6$ & $4.44 \lambda_{\mathrm{o}} \times 3.6 \lambda_{\mathrm{o}}$ & Greater than $\lambda_{\mathrm{o}} / 2$ & $15.6 \mathrm{dBi}$ & $18.4 \%$ & - \\
\hline 6 & {$[10]$} & $9.42-11.35$ & $2.20 \lambda_{\mathrm{o}} \times 2.20 \lambda_{\mathrm{o}}$ & $\approx \lambda_{\mathrm{o}} / 2$ & $12 \mathrm{dBi}$ & $18.56 \%$ & - \\
\hline 7 & {$[11]$} & $27.5-30$ & $2.98 \lambda_{\mathrm{o}} \times 2.98 \lambda_{\mathrm{o}}$ & $\approx \lambda_{\mathrm{o}} / 2$ & $15.5 \mathrm{dBi}$ & $8.6 \%$ & - \\
\hline 8 & {$[12]$} & $13.1-15.2$ & $3 \lambda_{\mathrm{o}} \times 3 \lambda_{\mathrm{o}}$ & $\approx \lambda_{\mathrm{o}} / 2$ & $13 \mathrm{dBi}$ & $15.5 \%$ & - \\
\hline 9 & {$[21]$} & $4.88-5.49$ & $1 \lambda_{\mathrm{o}} \times 1 \lambda_{\mathrm{o}}$ & $\approx \lambda_{\mathrm{o}} / 2$ & $8.5 \mathrm{dBi}$ & $11.88 \%$ & $2.88 \%$ \\
\hline 10 & This work & $4.93-5.89$ & $1 \lambda_{\mathrm{o}} \times 1 \lambda_{\mathrm{o}}$ & $\approx \lambda_{\mathrm{o}} / 2$ & $12.48 \mathrm{dBi}$ & $17.72 \%$ & $2.4 \%$ \\
\hline
\end{tabular}

Tab. 3. Performance comparison of antenna with RIS and FSS, and published literature. 


\section{Fabrication and Measured Results}

The fabrication of the pentagon shape patch antenna with the RIS surface is done on the FR4 substrate and the fabrication of the superstrate is done on the Rogers $\mathrm{RO}$ 4003C substrate by using LPKF milling machine S100. The superstrate is placed approximately half wavelength above the antenna using Teflon rods as shown in Fig. 8. Teflon rods are used which have no impact on radiation characteristics. The female type rounded four-hole coaxial RF SMA connector is inserted through FR4 substrates and it is soldered to the pentagon shape patch and the outer part is soldered to the ground layer. The reflection coefficient of the antenna is measured using a network analyzer in the frequency range of $4.5 \mathrm{GHz}$ to $6.5 \mathrm{GHz}$ as shown in Fig. 9a. The gain of the antenna is measured by using the gain transfer method in which the gain of a standard antenna is known.

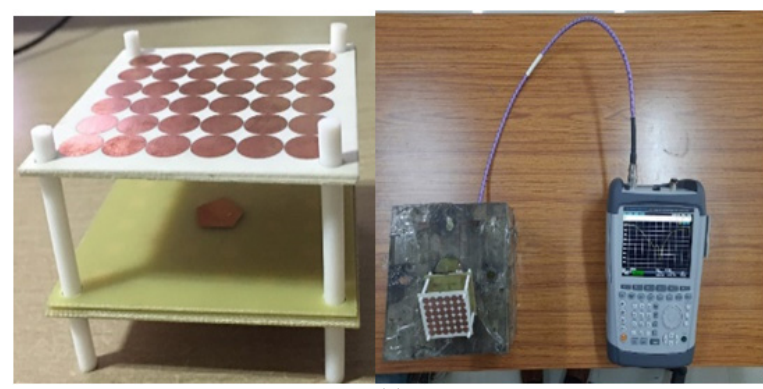

(a)
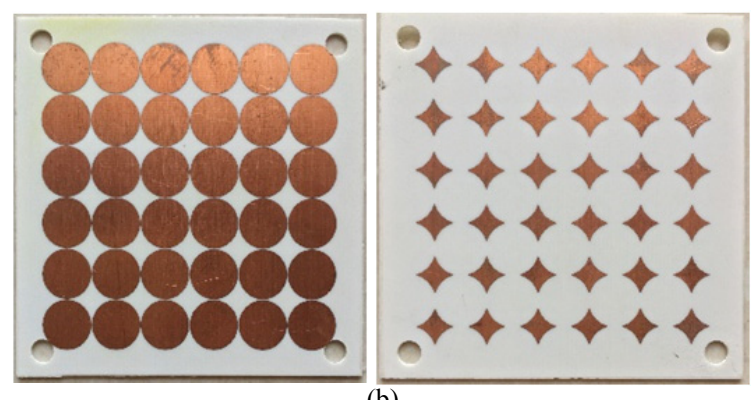

(b)

Fig. 8. Prototype of fabricated antenna with RIS and FSS: (a) Composite structure and its setup. (b) Capacitive layers, and inductive layers.

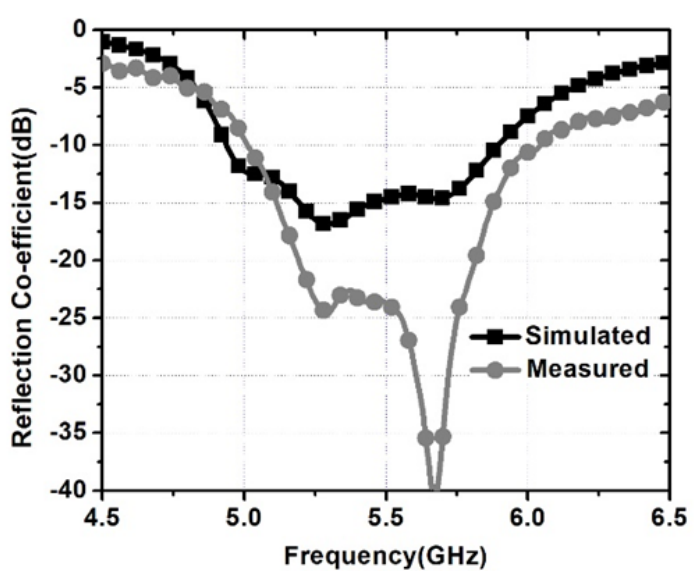

Fig. 9a. Simulated and measured reflection coefficient of antenna with RIS and FSS at $h=32 \mathrm{~mm}$.

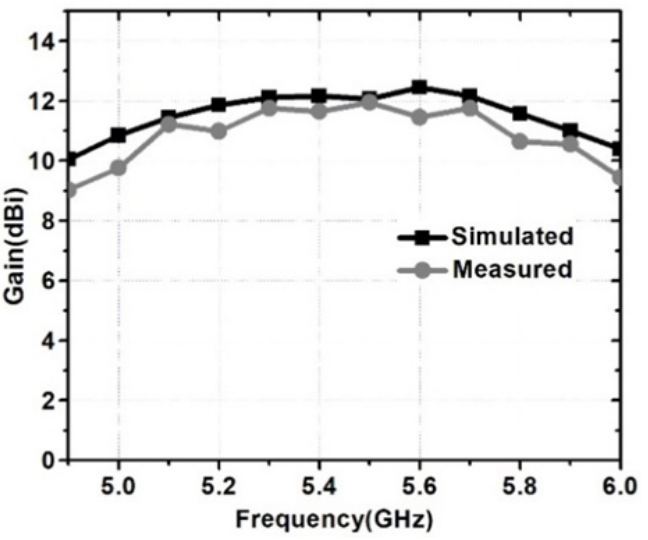

Fig. 9b. Simulated and measured gain of antenna with RIS and FSS at $h=32 \mathrm{~mm}$

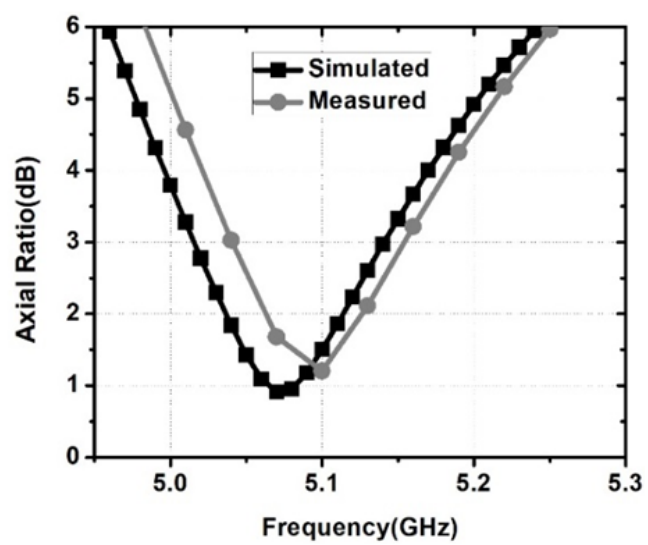

Fig. 9c. Simulated and measured axial ratio of antenna with RIS and FSS at $h=32 \mathrm{~mm}$.
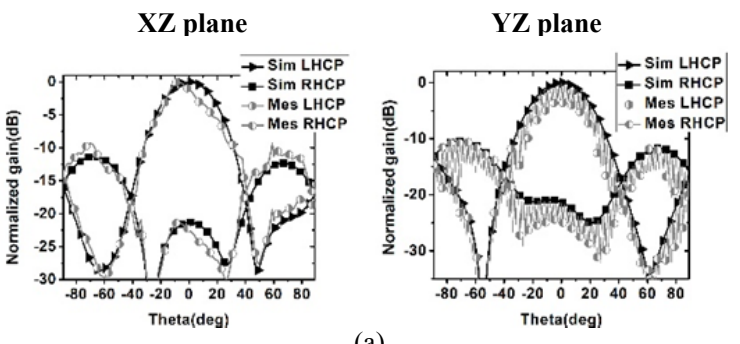

(a)
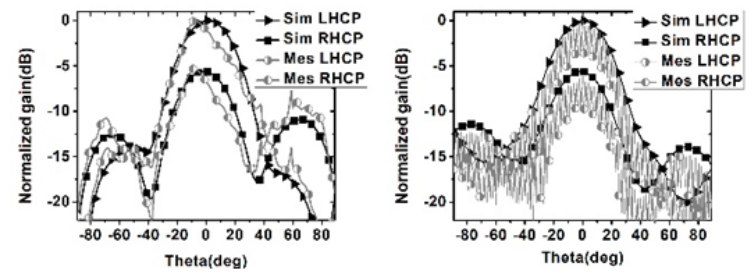

(b)
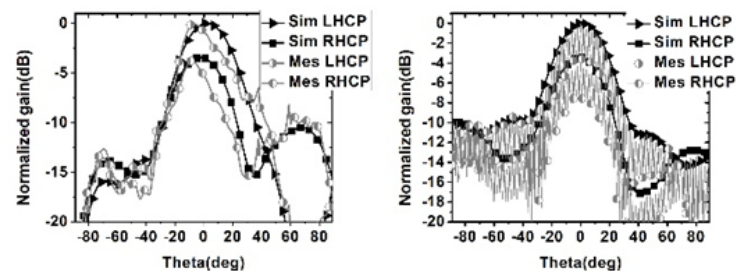

(c)

Fig. 10. Simulated and measured radiation patterns of antenna with RIS and FSS: (a) $5.1 \mathrm{GHz}$, (b) $5.5 \mathrm{GHz}$, (c) $5.8 \mathrm{GHz}$ 
In the setup, the standard horn antenna is used as a transmitting antenna, whose gain values are known. The fabricated antenna is used as a receiving antenna and it is rotated around its axis. The measured gain is similar to the simulated gain as shown in Fig. 9b. The axial ratio measurement is done and it is plotted in Fig. 9c. There is a slight change in the results due to fabrication errors. The radiation pattern of the fabricated antenna and simulated antenna are compared at $5.1 \mathrm{GHz}, 5.5 \mathrm{GHz}$, and $5.8 \mathrm{GHz}$ as shown in Fig. 10 .

\section{Conclusion}

The conventional pentagon shape patch antenna is designed. Highly reflective unit-cell characteristics are studied. The impedance bandwidth and gain of the antenna are increased simultaneously by incorporating additional structures like RIS structure as a ground plane to the conventional pentagon shape antenna and highly reflective FSS superstrate placed above the antenna. The composite structure of a pentagonal antenna with RIS and FSS acts like a Fabry Perot resonator. The proposed antenna achieves an impedance bandwidth of $17.72 \%(4.93 \mathrm{GHz}$ to $5.89 \mathrm{GHz}$ ) which is about a 10 percent improvement over the conventional antenna and a gain of $12.48 \mathrm{~dB}$ is obtained which is about a $9 \mathrm{~dB}$ improvement over the conventional antenna. The proposed antenna is improved both gain and bandwidth simultaneously at $5 \mathrm{GHz} \mathrm{Wi}-\mathrm{Fi}$ frequency in addition to that $3 \mathrm{~dB}$ axial ratio bandwidth of $2.4 \%(5.01 \mathrm{GHz}-5.14 \mathrm{GHz})$ is obtained. This antenna is fabricated and measurements are done in an anechoic chamber. The similarity between measured results and simulated results show that the antenna can be used for WLAN, Wi-Fi, and Wi-Max application.

\section{References}

[1] STUtZMAN, W. L., THIELE, G. A. Antenna Theory, and Design. 2nd ed. Hoboken (USA): Wiley, 1998. ISBN: 9780470576649

[2] YANG, W., WANG, H., CHE, W., et al. A wideband and highgain edge-fed patch antenna and array using artificial magnetic conductor structures. IEEE Antennas and Wireless Propagation. Letters, 2013, vol. 12, p. 769-772. DOI: 10.1109/LAWP.2013.2270943

[3] WANG, L., GUO, Y. X., SHENG, W. X. Wideband high-gain 60$\mathrm{GHz}$ LTCC L-probe patch antenna array with a soft surface. IEEE Transactions on Antennas and Propagation, 2013, vol. 61, no. 4 , p. 1802-1809. DOI: 10.1109/TAP.2012.2220331

[4] CHENG, Y. J., GUO, Y. X., LIU, Z. G. W-band large-scale highgain planar integrated antenna array. IEEE Transactions on Antennas and Propagation, 2014, vol. 62, no. 6, p. 3370-3373. DOI: $10.1109 /$ TAP.2014.2310483

[5] ORR, R., GOUSSETIS, G., FUSCO, V. Design method for circularly polarized Fabry-Perot cavity antennas. IEEE Transactions on Antennas and Propagation, 2013, vol. 62, no. 1, p. 19-26. DOI: 10.1109/TAP.2013.2286839
[6] PITRA, K., RAIDA, Z., LACIK, J. Low-profile circularly polarized antenna exploiting Fabry-Perot resonator principle. Radioengineering, 2015, vol. 24, no. 4, p. 898-905. DOI: $10.13164 /$ re.2015.0898

[7] CAO, W., LV, X., WANG, Q., et al. Wideband circularly polarized Fabry-Perot resonator antenna in Ku-band. IEEE Antennas and Wireless Propagation Letters, 2019, vol. 18, no. 4, p. 586-590. DOI: 10.1109/LAWP.2019.2896940

[8] AKBARI, M., GUPTA, S., SEBAK, A. R. High gain circularly polarized Fabry-Perot dielectric resonator antenna for MMW applications. In IEEE International Symposium on Antennas and Propagation (APSURSI). Puerto Rico, June 2016, p. 545-546. DOI: 10.1109/APS.2016.7695981

[9] ATTIA, H., ABDELGHANI, M. L., DENIDNI, T. A. Wideband and high-gain millimeter-wave antenna based on FSS Fabry-Perot cavity. IEEE Transactions on Antennas and Propagation, 2017, vol. 65, no. 10, p. 5589-5594. DOI: 10.1109/TAP.2017.2742550

[10] ZHENG, Y., GAO, J., ZHOU, Y., et al. Wideband gain enhancement and RCS reduction of Fabry-Perot resonator antenna with chessboard arranged metamaterial superstrate. IEEE Transactions on Antennas and Propagation, 2018, vol. 66, no. 2, p. 590-599. DOI: 10.1109/TAP.2017.2780896

[11] ASAADI, M., AFIFI, I., SEBAK, A. R. High gain and wideband high dense dielectric patch antenna using FSS superstrate for millimeter-wave applications. IEEE Access, 2018, vol. 6, p. 38243-38250. DOI: 10.1109/ACCESS.2018.2854225

[12] MERICHE, M. A., ATTIA, H., MESSAI, A., et al. Directive wideband cavity antenna with single-layer meta-superstrate. IEEE Antennas and Wireless Propagation Letters, 2019, vol. 18, no. 9, p. 1771-1774. DOI: 10.1109/LAWP.2019.2929579

[13] LEE, Y. J., YEO, J., MITTRA, R., et al. Design of a highdirectivity electromagnetic bandgap (EBG) resonator antenna using a frequency-selective surface (FSS) superstrate. Microwave and Optical Technology Letters, 2004, vol. 43, no.6, p. 462-467. DOI: $10.1002 / \mathrm{mop} .20502$

[14] HOSSEINI, A., CAPOLINO, F., DE FlAVIIS, F. Gain enhancement of a V-band antenna using a Fabry-Perot cavity with a self-sustained all-metal cap with FSS. IEEE Transactions on. Antennas and Propagation, 2015, vol. 63, no. 3, p. 909-921. DOI: 10.1109/TAP.2014.2386358

[15] LAU, K. L., LUK, K. M. A novel wide-band circularly polarized patch antenna based on L-probe and aperture-coupling techniques. IEEE Transactions on Antennas and Propagation, 2005, vol. 53, no. 1 , p. 577-580. DOI: 10.1109/TAP.2004.838796

[16] LAU, K. L., LUK, K. M. A wide-band circularly polarized Lprobe coupled patch antenna for dual-band operation. IEEE Transactions on Antennas and Propagation, 2005, vol. 53, no. 8, p. 2636-2644. DOI: 10.1109/TAP.2005.851818

[17] KIM, S. M., YANG, W. G. Single feed wideband circular polarized patch antenna. Electronic Letters, 2007, vol. 43, no. 13, p. 703-704. DOI: 10.1049/el:20070677

[18] SIEVENPIPER, D., ZHANG, L., BROAS, R. F. J., et al. High impedance electromagnetic surfaces with a forbidden frequency band. IEEE Transactions on Microwave Theory and Techniques. 1999, vol. 47, no. 11, p. 2059-2074. DOI: 10.1109/22.798001

[19] ZHANG, Y., VON HAGEN, J., YOUNIS, M., et al. Planar artificial magnetic conductors and patch antennas. IEEE Transactions on Antennas and Propagation, 2003, vol. 51, no. 10, p. 2704-2712. DOI: 10.1109/TAP.2003.817550

[20] NAKAMURA, T., FUKUSAKO, T. Broadband design of circularly polarized microstrip patch antenna using artificial ground structure with rectangular unit cells. IEEE Transactions on Antennas and Propagation, 2011, vol. 59, no. 6, p. 2103-2110. DOI: 10.1109/TAP.2011.2143656 
[21] SRINIVAS, G., SARMA, N. V. S. N., VAKULA, D. Gain and bandwidth improvement of circularly polarized pentagonal patch antenna. In TEQIP III Sponsored International Conference on Microwave Integrated Circuits, Photonics and Wireless Networks $(I M I C P W)$. Trichy (India), 2019, p. 274-277. DOI: 10.1109/IMICPW.2019.8933229

[22] BAI, B. K., THAKUR, A. Pentagonal shaped microstrip patch antenna in wireless capsule endoscopy system. In International Conference on Information Technology Convergence and Services, Software Engineering and Applications, Signal and Image Processing, Computer Science \& Information Technology 04. 2012, p. 47-54. DOI: $10.5121 /$ csit.2012.2105

\section{About the Authors ...}

Guthi SRINIVAS is born in Telangana, India in 1989. He received the bachelor's degree in Electronics and Communication Engineering from Jawaharlal Nehru Technical University, Andhra Pradesh, India, and the master's degree in Communications Systems from the NIT Allahabad, Uttar Pradesh, India, with a focus on MIMO antennas in 2012 and 2014, respectively. From 2014 to 2017, he worked as an Assistant Professor at Gurunanak Institute of Technical Campus, Telangana. Since 2017, he is pursuing a Ph.D. degree in the Department of Electronics and Communication Engineering, NIT Warangal, Telangana, India. His research area is gain and bandwidth improvement of the antenna.

Damera VAKULA received the bachelor's degree in Electronics and Communication Engineering from Nagarjuna University, Andhra Pradesh, India, and the master's degree in Tech from the Birla Institute of Technology, Mesra, India, with a focus on microwave specialization in 1992 and 1994, respectively, and the Ph.D. degree in Fault Diagnostics of Antenna Arrays from the National Institute of Technology, Warangal, India, in 2010. She has been an Assistant Professor with the National Institute of Technology since 2006. She has authored 31 papers in international conferences and journals. Her areas of interests include phase array antennas, ultra wideband antennas, multiband antennas, fault diagnostics, and neural network. 